\section{O USO DA INTELIGÊNCIA COLETIVA E O MÉTODO SIMPLEX NA BUSCA DE MINIMIZAR CUSTO UTILIZANDO DISPOSITIVOS MÓVEIS}

\section{USE OF COLLECTIVE INTELLIGENCE AND SIMPLEX METHOD IN SEARCH MINIMIZE COST USING MOBILE DEVICES}

\author{
Adilson José da Cruz ${ }^{1}$; Francisco Assis da Silva²; Francisco Virginio \\ Maracci $^{2}$; Mário Augusto Pazoti ${ }^{2}$ \\ Discente da Faculdade de Informática da UNOESTE ${ }^{1}$; Docente da Faculdade de \\ Informática da UNOESTE ${ }^{2}$.
}

RESUMO - Este trabalho apresenta uma solução de software para dispositivos móveis capaz de auxiliar um consumidor a fazer economia durante o processo de compras, permitindo que se possa ter uma noção da variação de preços antes de se dirigir ao local de compra, contribuindo desta forma para a tomada de decisão. Com o aplicativo, o consumidor cria listas de produtos, atualiza e compartilha preços, permitindo que outros consumidores tenham conhecimento sobre estas informações, e realiza cálculo de menor custo da lista de compras utilizando seu dispositivo móvel. Para o cálculo de menor custo foi utilizado o método Simplex, utilizado na Programação Linear para cálculo de solução ótima, minimizando custos. Para testar a funcionalidade do aplicativo foram realizados testes informando uma lista de produtos da cesta básica e foram realizados cálculos de menor custo levando em consideração o valor total da lista em vários estabelecimentos.

Palavras-chave: Inteligência Coletiva; Método Simplex; Programação Linear; Crowdsourcing; Dispositivos Móveis.

ABSTRACT - This paper presents a software solution for mobile devices to help a consumer to save during the shopping process, allowing getting a sense of price variation before to go the place of purchase, so contributing to decision-making. Through the application, the consumer creates lists of products, updates and shares prices, allowing other consumers are aware about this information, and it performs calculation of lower cost of the shopping list using your mobile device. Simplex Method was used to calculate the lowest cost also used in Linear Programming to calculate the optimal solution, minimizing costs. Tests with application functionality have been made to supply a list of basic food products and lower cost calculations were made taking into account the total value of the list in various establishments. KEYWORDS: Collective Intelligence; Simplex Method; Linear Programming; Crowdsourcing; Mobile Devices.
Recebido em: 13/06/2014 Revisado em: 26/09/2014 Aprovado em: 07/10/2014 


\section{INTRODUÇÃO}

A pesquisa de preços sempre foi algo muito comum no cotidiano dos consumidores brasileiros. A economia brasileira com suas altas taxas de inflação e juros faz com que o preço de produtos e serviços tenha grande variação em diversas localidades do país. Segundo BBC Brasil (2013), a inflação é um velho inimigo da economia brasileira, em que o aumento dos preços mina o poder de compra da população, especialmente das classes mais baixas que, com menos dinheiro no bolso, correm mais riscos de ficar endividada e inadimplente.

Com a evolução dos meios de comunicação, em especial a Internet, a informação ficou muito mais acessível e também alterou a maneira como as pessoas realizam pesquisa de preços. Foram desenvolvidas ferramentas, como sistemas especializados em pesquisa de preços de diversos tipos de produtos, em que facilmente se podem encontrar ofertas e produtos, servindo como base para a decisão de compra do consumidor, que deste modo realiza economia nas compras, sejam estas feitas de forma presencial nas lojas, ou virtual com o uso da Internet.

A telefonia também acompanhou a evolução dos meios de comunicação, e os celulares que antes serviam apenas para comunicação de voz se tornaram máquinas capazes de fotografar, enviar mensagens de texto, acessar a Internet etc. A partir desta evolução surgiram então os Smartphones, os "telefones inteligentes", que são híbridos entre celular e computador e possuem funcionalidades que vão além do recebimento de chamadas. Eles não possuem o hardware potente de um computador, mas não são tão simples como o celular comum, pois englobam algumas das principais tecnologias de comunicação: Internet, GPS, e-mail, SMS etc. Além disso, os Smartphones utilizam Sistema Operacional, como os computadores, e permitem que neles sejam instalados aplicativos estendendo suas funcionalidades (TECHTUDO, 2011).

O uso do Smartphone acabou desenvolvendo novos hábitos na população que o utiliza, não apenas para se comunicar por meio de voz, mas também para enviar mensagens de texto, fotografar, pesquisar e acessar conteúdos da internet, jogar, ouvir música, se orientar, localizar endereços utilizando dados de GPS etc (BUENO, 2013).

Uma das principais utilizações do Smartphone no processo de compra é a pesquisa de produtos e preços. Nos casos em que o usuário utilizou o Smartphone para realizar uma pesquisa, em $46 \%$ das vezes ele acabou não comprando o produto. Por outro lado, $45 \%$ dos donos de Smartphones realizaram a compra por meio de um computador depois de fazer uma pesquisa 
móvel, e $27 \%$ fizeram a compra diretamente pelo Smartphone. Outros $30 \%$ acabaram fazendo a compra de forma off-line, dirigindo-se à loja física depois de fazer a pesquisa no dispositivo (OUR PLANET MOBILE, 2013).

Os dados sobre a influência dos Smartphones na decisão de compra são bem semelhantes: $31 \%$ dos usuários levam seus Smartphones às lojas para pesquisar produtos. Outros $29 \%$ chegaram a mudar de ideia quanto às compras na loja física após realizarem uma pesquisa no Smartphone, e $30 \%$ mudaram de ideia quanto às compras on-line por terem buscado informações na Internet com seus aparelhos móveis (OUR PLANET MOBILE, 2013).

Utilizando a Internet como meio, a câmera do Smartphone, as mensagens de texto, juntamente com a mobilidade dos Smartphones e dispositivos móveis, pode-se produzir e distribuir informação em tempo real. Algo acontece e instantaneamente já pode ser registrado por alguém e distribuído de forma que diversas outras pessoas, que tenham acesso à tecnologia, possam ficar sabendo do ocorrido em minutos. Ficou mais fácil se comunicar, produzir e distribuir informação (HOWE, 2009).

Em seus estudos, Levy (2007) cita que o fato de se poder trocar informações, de interagir com outras pessoas pelos meios de comunicação, traz um aprendizado recíproco.
Ele define essa interação como Inteligência Coletiva: "É uma inteligência distribuída em toda parte, incessantemente valorizada, coordenada em tempo real, que resulta em uma mobilização efetiva das competências".

Desta evolução dos meios de comunicação surgiu o Crowdsourcing, método que se utiliza da inteligência e conhecimentos coletivos para resolver problemas, criar conteúdo, soluções ou desenvolver tecnologia. É um modelo de solução compartilhada de problemas através de uma rede de solucionadores, que normalmente se encontram em forma de comunidades que possuem um interesse em comum. Os problemas são transmitidos ao grupo em forma de convite e estes propõem soluções para estes problemas e também classificam outras soluções procurando as melhores (HOWE, 2009).

A proposta deste trabalho é apresentar um novo aplicativo, que utilizando a mobilidade dos dispositivos móveis possa informar às pessoas sobre os preços dos produtos nos estabelecimentos e realizar o cálculo de menor custo de uma lista de produtos relacionada pelo usuário do aplicativo, demonstrando onde poderá ser realizada a compra de menor custo. Para isso, vários fatores são considerados para o cálculo, tais como a quantidade de produtos a serem comprados e a distância do 
estabelecimento de compra em relação à localização do usuário.

O aplicativo foi idealizado tendo como base o modelo Crowdsourcing, em que os usuários podem cadastrar, atualizar, criar listas e publicar os preços dos produtos em uma base disponível na Web. O aplicativo permite a interação dos usuários de modo que os dados da base sempre sejam atualizados e consultados por eles.

Com o uso da câmera do Smartphone, os códigos de barras dos produtos são lidos e os códigos são usados para cadastro de um novo produto ou para atualização de preço. Se não houver o cadastro do estabelecimento, este também pode ser feito com o uso do aplicativo. $O$ intuito é que esse aplicativo colabore com a economia em compras e com a divulgação dos preços dos produtos nos estabelecimentos, permitindo que se possa ter uma noção da variação de preços antes de se deslocar para o local de compra e, possa desse modo contribuir para a tomada de decisão do consumidor.

Para o cálculo de menor custo foi utilizado o Método Simplex que é uma técnica utilizada para determinar, numericamente, a solução ótima de um modelo de Programação Linear. Busca-se com o método soluções para problemas onde se deseja alcançar o lucro máximo ou o custo mínimo, em situações nas quais se tem diversas opções de escolha sujeitas a algum tipo de restrição ou regulamentação (OKIISHI; SOUZA, 2013).

O aplicativo foi desenvolvido para a plataforma de dispositivos móveis Android, por ser uma das mais populares do mercado e permitir o desenvolvimento rápido de aplicações.

As demais seções deste trabalho estão organizadas da seguinte maneira: na Seção 2 são apresentados os trabalhos relacionados com a mesma linha de pesquisa; na Seção 3 é apresentado o método Simplex e a modelagem utilizada para a formulação do problema a ser otimizado pelo método; na Seção 4 é apresentado o aplicativo implementado neste trabalho; na Seção 5 são apresentados os experimentos utilizados para avaliar a eficiência do método empregado para calcular a otimização de custos assim como os resultados obtidos com os experimentos, e por fim, na Seção 6 são apresentadas as conclusões e considerações finais do trabalho.

\section{TRABALHOS RELACIONADOS}

Nesta seção são apresentados alguns trabalhos e aplicativos que aplicam o conceito de inteligência coletiva, seja para a comparação de preços ou para outros fins, a fim de reduzir custos.

No trabalho de Ceccatto (2012), o conceito de inteligência coletiva foi aplicado na construção de um aplicativo para o 
fornecimento de informações de determinados tipos de produtos como promoções, descontos, liquidações, divulgados por escolha das empresas parceiras do projeto. O autor aplica os conceitos de inteligência coletiva por meio do compartilhamento de informações que os usuários podem fazer ao comparar ofertas semelhantes, listar a mais vantajosa e poder compartilhar essas informações na rede social Facebook, assim fazendo a divulgação não só do produto como também das empresas fornecedoras do mesmo.

O aplicativo "Touch Economia" (GOOGLE PLAY, 2014), foi desenvolvido pelo Instituto Curitiba de Informática, e apresenta a comparação de preços entre supermercados da cidade de Curitiba, pela Secretaria Municipal de Abastecimento. O aplicativo é um comparador de preços, em que o cidadão pode verificar qual mercado oferece o melhor custo (Figura 1). A comparação pode ser feita para um item ou uma lista completa. De acordo com $\mathrm{ICl}$ (2012), o diferencial do aplicativo é que a captação de preços é feita mediante pesquisa presencial, ao invés de automaticamente pela internet. Assim, podem ser encontradas diferenças de até $40 \%$ de preço entre um mercado e outro, mesmo dentro da mesma rede de lojas.

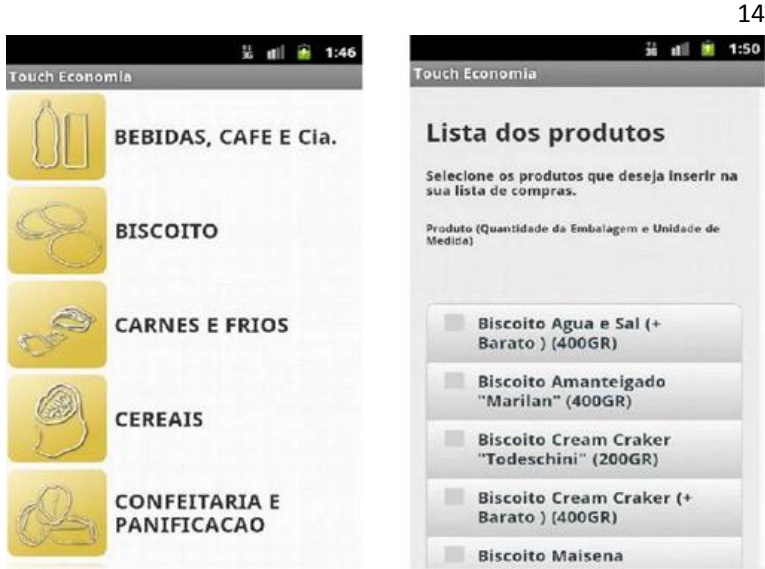

Figura 1 - Interface Aplicativo Touch Economia.

O "Boa lista" é outro aplicativo móbile que permite a criação de listas de compras de forma simples, permitindo que o usuário faça - escaneamento do código de barras, utilizando a câmera do celular, para localizar o produto da compra ou permitir o seu cadastramento (Figura 2). A aplicação permite a comparação de preços em lojas online e físicas. Sua base de dados pode ser alimentada pelos próprios usuários do aplicativo. Pode ser instalado em Smartphones com sistema operacional Android da versão 2.1 em diante (BOA LISTA, 2014). A Figura 2 mostra a interface do aplicativo.
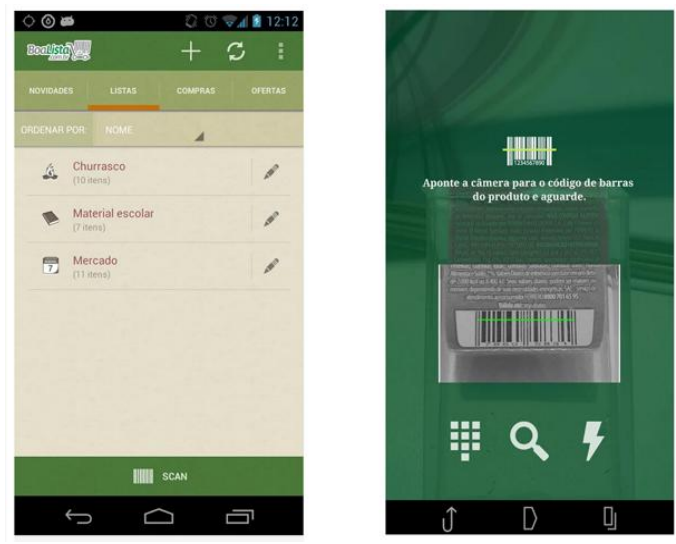

Figura 2-Interface do Aplicativo Boa Lista, que permite escanear 0 código de barras dos produtos. 
Por fim, o "meu carrinho" é um aplicativo que permite a criação e gerenciamento de listas de compras. Possui integração com a Web, permitindo ao usuário que monte suas listas com o uso do site e pode obtê-las via celular. O usuário pode escanear o código de barra dos produtos para obter a relação de ofertas. Funciona de modo off-line, não precisando estar conectado a uma rede $3 \mathrm{G}$ ou Wi-Fi para checar as listas no supermercado e montá-las com itens sem uma marca definida. Possui integração com o Buscapé, serviço de comparação de preços da Internet (BUSCAPÉ COMPANY, 2014). A Figura 3 mostra a interface do aplicativo.
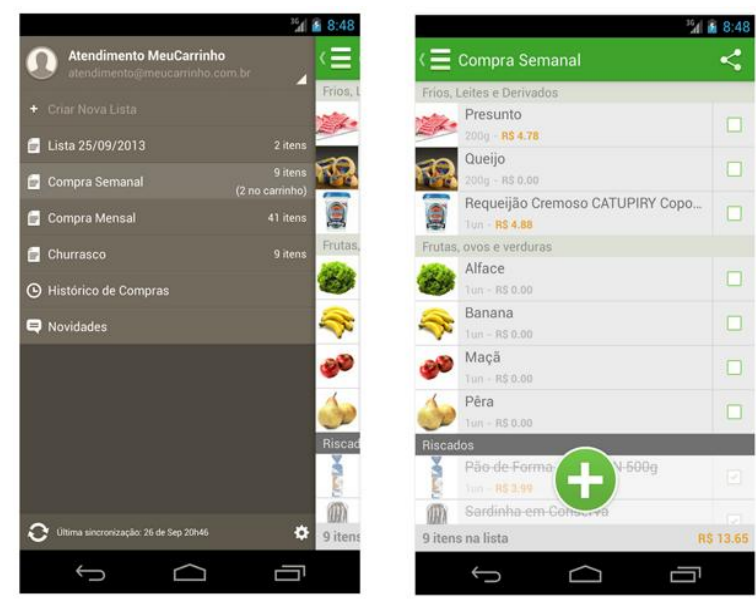

Figura 3.Interface do Aplicativo Meu Carrinho.

\section{O MÉTODO SIMPLEX}

O Método Simplex foi desenvolvido em 1947 por George B. Dantzig para a solução de problemas de Programação Linear onde se procura aumentar ou diminuir ao máximo um determinado valor de acordo com diversas necessidades e restrições. A
Programação Linear $(\mathrm{PL})$ é uma área da pesquisa operacional com vasta aplicação em apoio à decisão. A Programação Linear consiste em uma programação matemática onde a função-objetivo e suas restrições assumem caracteristicas lineares, possuindo variadas aplicações na gestão gerencial, como por exemplo, a administração de produção, análise de investimentos, logística empresarial, problemas de transportes, problemas onde se busca a utilização ótima dos recursos disponíveis considerando-se as limitações impostas pelo processo produtivo ou pelo mercado (LACHTERMACHER, 2002).

A palavra "Programação" na definição do conceito de Programação Linear, diz respeito a planejamento e não a programação de computadores (OKIISHI; SOUZA, 2013).

Para iniciar o Método Simplex necessita-se de uma solução básica viável inicial, a qual é um dos pontos extremos do conjunto de soluções compatíveis (ou viáveis ou factíveis) do modelo de Programação Linear. Este método verifica se a presente solução é ótima. Se esta não for é porque um dos demais pontos extremos adjacentes (vértices) fornecem valor menor para a função objetivo, quando o problema considerado é de minimização. Então se faz uma mudança de vértice na direção que mais diminua a função objetivo e verifica se este novo vértice é ótimo. O processo termina 
quando estando em um ponto extremo, todos os outros pontos extremos adjacentes fornecem valores maiores para a função de minimização.

Alguns passos para 0 desenvolvimento do modelo são:

- Identificar quais decisões efetivamente resolvem o problema;

- Definir variáveis de decisão;

- Identificar quais as restrições que limitam as decisões a tomar;

- Definir o conjunto de equações ou inequações;

- Definir objetivos capazes de indicar que uma decisão é preferível a outras;

- Definir uma função objetivo.

Em geral o modelo matemático a ser otimizado tem o formato apresentado nas Equações (1).

$\min ($ ou $\max ) z=c_{1} x_{1}+c_{2} x_{2}+\ldots+c_{n} x_{n}$ sujeito $a$ :

$$
\begin{aligned}
& a_{11} x_{1}+a_{12} x_{2}+\ldots+a_{1 n} x_{n} \sim b_{1} \\
& a_{22} x_{1}+a_{22} x_{2}+\ldots+a_{2 n} x_{n} \sim b_{2} \\
& \quad \vdots \\
& a_{m 1} x_{1}+a_{m 2} x_{2}+\ldots+a_{m m} x_{n} \sim b_{m} \\
& x_{1}, x_{2}, \ldots x_{n} \geq 0, \text { e int eiras } \\
& \text { onde } \sim \text { pode ser } \geq, \leq, \text { ou }=
\end{aligned}
$$

No problema, a função objetivo será a função Custo, onde se deseja realizar a minimização do custo da lista de compras, e as variáveis de decisão $(x 1, x 2, x 3 \ldots)$ serão o quantidade de cada produto a ser comprado, sujeito as restrições de quantidade mínima para cada um deles.

Aplicando o conceito do Método Simplex, foi criado um modelo para solucionar o problema apresentado neste trabalho, que é a redução de custos em compras.

A Tabela 1 mostra a simulação da compra de alguns produtos em lojas onde há variação de preços para os produtos e a distância das lojas em relação a localização do usuário do aplicativo são diferentes. 
Tabela 1. Modelo utilizado para simular o cálculo de menor custo utilizado pelo aplicativo desenvolvido neste trabalho.

\begin{tabular}{|c|c|c|c|c|c|c|c|}
\hline & \multicolumn{2}{|c|}{ Loja 1} & \multicolumn{2}{|c|}{ Loja 2} & \multicolumn{2}{|c|}{ Loja 3} & $\begin{array}{c}\text { Quantidade do } \\
\text { Produto }\end{array}$ \\
\hline Produto A (x1) & $\mathrm{R} \$$ & 8,50 & $\mathrm{R} \$$ & 9,00 & $\mathrm{R} \$$ & 7,50 & 5 \\
\hline Produto B (x2) & $\mathrm{R} \$$ & 2,90 & $\mathrm{R} \$$ & 1,00 & $\mathrm{RS}$ & 3,25 & 7 \\
\hline Produto C (x3) & $\mathrm{R} \$$ & 4,80 & $\mathrm{R} \$$ & 2,90 & $\mathrm{R} \$$ & 3,00 & 2 \\
\hline Distâncias (x4) & & & & & & $\mathrm{Km}$ & - \\
\hline
\end{tabular}

Definindo $x 1$ como a quantidade do produto $A, x 2$ a quantidade do produto $B$ e $x 3$ a quantidade do produto $C$, definiu-se a seguinte função objetivo:

Minimizar $Z=8.5 \times 1+2.9 \times 2+4.8 \times 3 \quad(2)$

A função objetivo usada neste modelo, destinada a redução do custo, foi aplicado sobre o preço dos produtos em cada loja, verificando no final em qual é mais vantajoso comprar:

ou seja: Quantidade do Produto A* Valor do Produto A na Loja $1+$ Quantidade do Produto B * Valor do Produto B na Loja 1 + Quantidade do Produto $C *$ Valor do Produto C na Loja 1.

Sujeito as restrições de quantidade mínima do produto que deve ser comprado

Deve-se comprar no mínimo 5 unidades do Produto A, 7 unidades do Produto B e 2 unidades do Produto $C$, se apresentando da seguinte forma:

$$
\begin{aligned}
& X 1>=5 ; \\
& X 2>=7 ;
\end{aligned}
$$

A distância é um recurso que é somado ao custo total da lista calculado pelo método.

\section{DESCRIÇÃO DO APLICATIVO}

O aplicativo construído, Lista Fácil, utiliza um WebService $^{1}$ desenvolvido na linguagem .Net, que se comunica com uma base de dados gerenciada pelo Gerenciador de Banco de Dados SQL Server 2005. Este WebService é responsável por fazer a integração do aplicativo móvel com a Web, como é ilustrado na Figura 4. Com o uso do aplicativo móvel o usuário cria sua lista, pesquisa e atualiza preço de produtos nos estabelecimentos comerciais cadastrados e faz o cálculo de menor custo da lista de compras. Estes dados são enviados pelo aplicativo ao WebService que processa as informações, atualiza a base de dados e

\footnotetext{
${ }^{1}$ Web Service: é uma solução utilizada na integração de sistemas e na comunicação entre aplicações diferentes. Com esta tecnologia é possível que novas aplicações possam interagir com aquelas que já existem e que sistemas desenvolvidos em plataformas diferentes sejam compatíveis.
} 
retorna o resultado do cálculo de menor custo da lista para o aplicativo Lista Fácil.

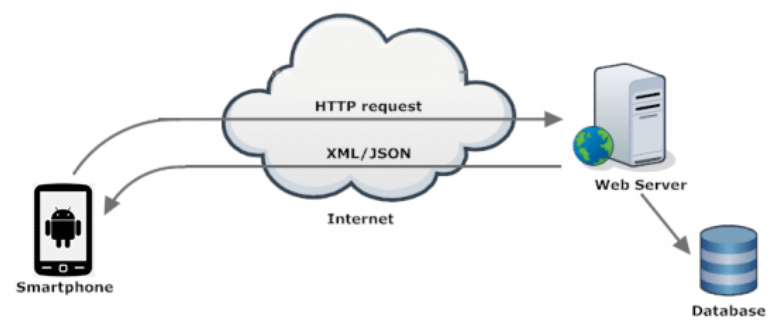

Figura 4. Modelo de Integração do Aplicativo. Esta figura demonstra como funciona o fluxo de dados entre o dispositivo móvel, com o aplicativo Lista Fácil, o WebService e o banco de dados que se encontra na Internet.

O aplicativo se comunica com o WebService enviando dados para o cadastro de novos produtos, estabelecimentos, ofertas. O WebService recebe estas requisições e processa persistindo os dados no banco de dados. O WebService também recebe a solicitação de cálculo de menor custo. Para isto o aplicativo envia a lista de produtos que deseja consultar e o WebService processa essa consulta e retorna para o aplicativo como resultado os locais onde a compra dos produtos tem menor custo.

Para o cálculo de distância entre a cidade de consulta e a cidade da loja o WebService utiliza o Google Distance Matrix API, um serviço que calcula rotas entre localidades utilizando uma solicitação $\mathrm{HTTP}^{2}$, que a partir da informação da cidade de

\footnotetext{
${ }^{2}$ HTTP: Hypertext Transfer Protocol ,em português Protocolo de Transferência de Hipertexto, é um protocolo de comunicação, utilizado para sistemas de informação de hipermídia, distribuídos e colaborativos (TANENBAUM, 2003).
}

origem e cidade de destino retorna a distância total e tempo de deslocamento. A requisição HTTP da API de Matriz de distâncias tem o seguinte formato:

http://maps.googleapis.com/maps/api/distancemat rix/output?parameters

O "output", ou seja o retorno de dados, da API pode ser json (recomendado), indica a saída em JSON (JavaScript Object Notation); ou xml, que indica a saída como XML. Na Figura 5 tem-se o exemplo da solicitação de cálculo realizada, exibida no navegador, das informações de distância entre Bastos, SP e Presidente Prudente, SP. A requisição HTTP utilizada foi: http://maps.googleapis.com/maps/api/dista ncematrix/xml?origins=Bastos+SP\&destinatio ns=Presidente+Prudente $\&$ sensor=false

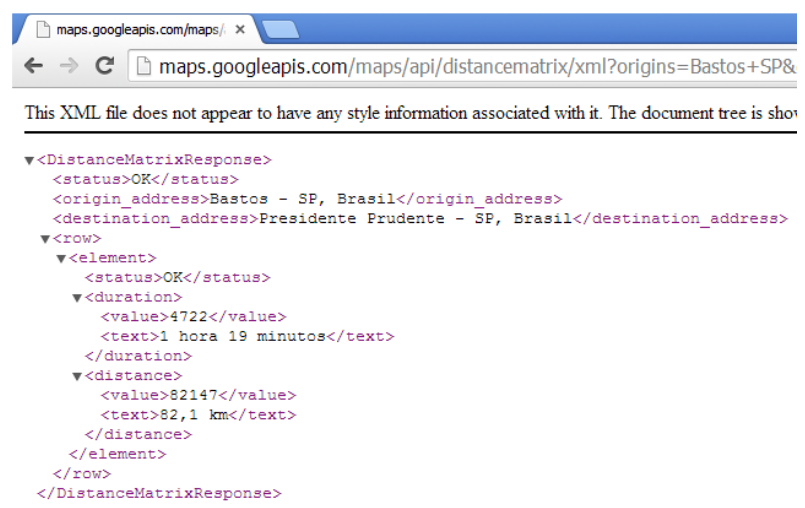

Figura 5. Retorno resultante da consulta de distância entre Bastos, SP e Presidente Prudente, SP, utilizando o Google Distance Matrix API.

Acessando a tela principal do Lista Fácil serão exibidas as listas criadas, com suas respectivas quantidades, como mostrado na Figura 6. O usuário poderá criar novas listas e incluir seus produtos. 


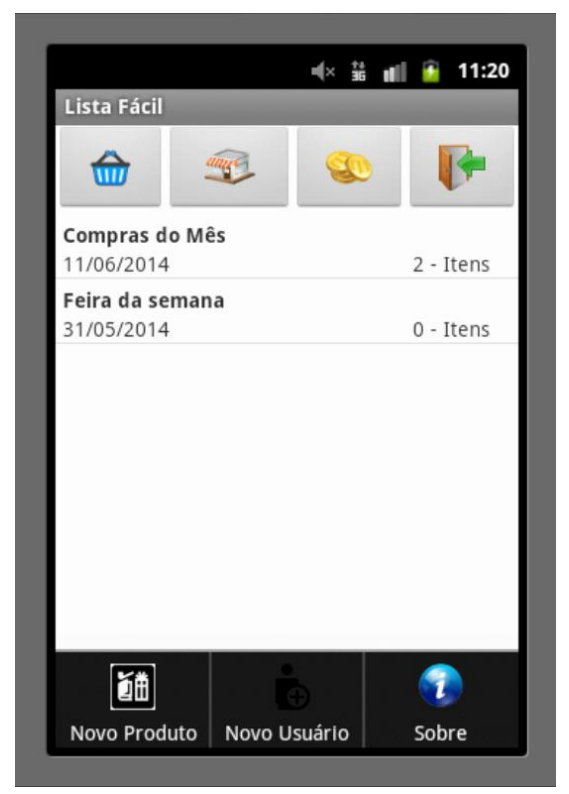

Figura 6. Tela inicial do aplicativo Lista Fácil em conjunto com a tela de demonstra os detalhes de uma das listas criadas.

Pode-se pesquisar 0 preço dos produtos que estão cadastrados na base de dados e fazer a atualização do preço, como demonstrado na Figura 7. A pesquisa do produto também pode ser realizada por código de barras.

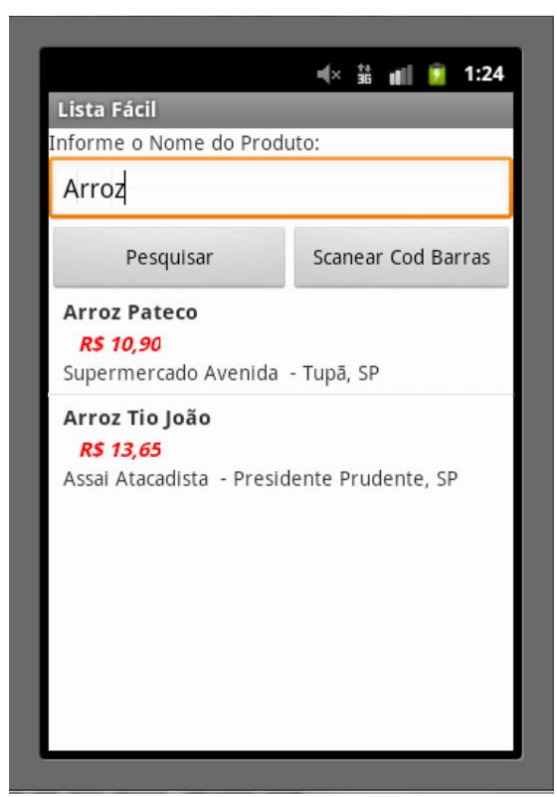

Figura 7. Telas onde são realizadas as pesquisas de produtos e atualização de preços.
O modelo de dados utilizado pelo aplicativo Lista Fácil é apresentado na Figura 8. Este modelo representa como as informações estão organizadas de maneira lógica, e são armazenadas, dentro do aplicativo e da base de dados presente na Web. Utilizando este modelo, o aplicativo armazena e recupera informações sobre os estabelecimentos comerciais, produtos, listas criadas pelo usuário, cotação dos produtos, histórico de cálculos realizados pelo usuário e informações sobre o próprio usuário como login e senha utilizados para realizar o cadastro de cotações para os produtos. 


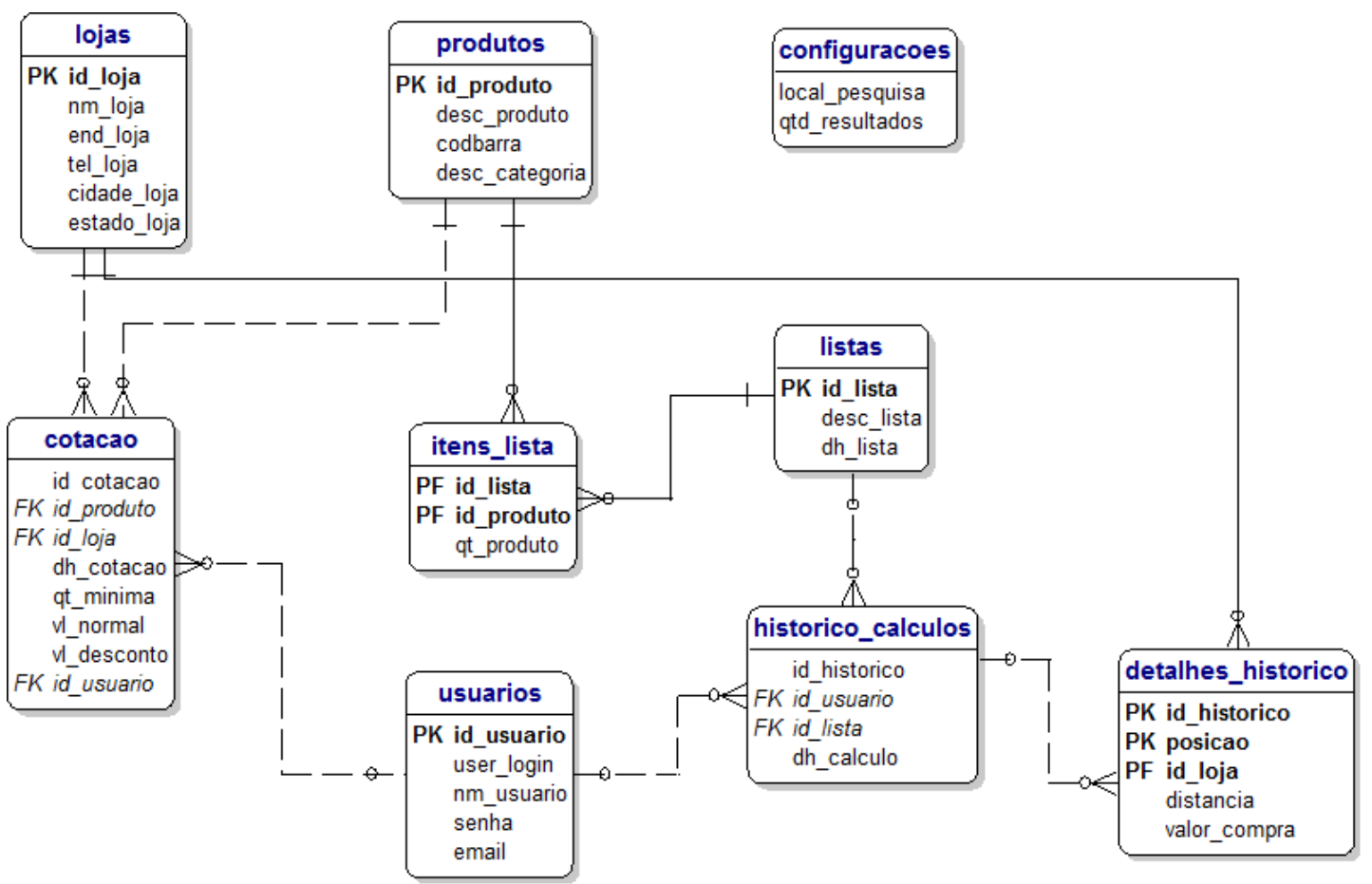

Figura 8. Modelo de dados utilizado pelo aplicativo Lista Fácil.

Foi utilizado no projeto do aplicativo Lista Fácil a biblioteca Zxing (ZXING, 2014), que permite o processamento de imagem de código de barras. Zxing é uma biblioteca de código aberto, ou seja pode ser utilizada gratuitamente, desenvolvida em Java e capaz de portar à muitas outras linguagens a funcionalidade de leitura de código de barras.

\section{EXPERIMENTO REALIZADO}

Foi criada uma lista com 15 produtos da cesta básica. Foram colhidas informações sobre estes produtos em 5 estabelecimentos diferentes com distâncias variadas. A distância foi calculada tendo como origem a cidade de Bastos, interior do Estado de São Paulo.

Para os testes de funcionalidade do aplicativo Lista Fácil, uma lista com a quantidade de produtos foi criada, com seus devidos preços atualizados, como mostra a Tabela 2. Foram realizadas diversas simulações e ajustes no algoritmo para que o cálculo pudesse se adequar ao objetivo proposto que é a redução de custos na compra. 
Tabela 2- Tabela de 15 produtos da cesta básica utilizados para a simulação.

\begin{tabular}{|c|c|c|c|c|c|c|}
\hline Produtos & $\begin{array}{c}\text { Supermercado } \\
\text { Kawakami } \\
\text { Bastos/SP }\end{array}$ & $\begin{array}{c}\text { Supermercado } \\
\text { Avenida } \\
\text { Rancharia/SP }\end{array}$ & $\begin{array}{c}\text { Assai } \\
\text { Atacadista } \\
\text { Presidente } \\
\text { Prudente/SP }\end{array}$ & $\begin{array}{c}\text { Supermercado } \\
\text { Avenida } \\
\text { Tupã/SP }\end{array}$ & $\begin{array}{c}\text { Cocipa } \\
\text { Inúbia } \\
\text { Paulista/SP }\end{array}$ & $\begin{array}{l}\text { Quantidade de } \\
\text { Produtos }\end{array}$ \\
\hline Arroz $5 \mathrm{Kg}$ & $\mathrm{R} \$ 11,80$ & $\mathrm{R} \$ 13,15$ & $\mathrm{R} \$ 10,78$ & $\mathrm{R} \$ 11,45$ & $\mathrm{R} \$ 10,19$ & 3 \\
\hline Feijão $1 \mathrm{Kg}$ & $\mathrm{R} \$ 2,59$ & $\mathrm{R} \$ 3,95$ & $\mathrm{R} \$ 4,12$ & $\mathrm{R} \$ 2,90$ & $\mathrm{R} \$ 4,13$ & 2 \\
\hline Macarrão 500g & $\mathrm{R} \$ 2,17$ & $\mathrm{R} \$ 2,99$ & $\mathrm{R} \$ 2,55$ & $\mathrm{R} \$ 2,67$ & $\mathrm{R} \$ 2,48$ & 3 \\
\hline Leite Integral 11 & $\mathrm{R} \$ 2,89$ & $\mathrm{R} \$ 2,18$ & $\mathrm{R} \$ 2,32$ & $\mathrm{R} \$ 2,55$ & $\mathrm{R} \$ 3,10$ & 24 \\
\hline Café 500g & $\mathrm{R} \$ 5,48$ & $\mathrm{R} \$ 5,99$ & $\mathrm{R} \$ 5,38$ & $\mathrm{R} \$ 5,67$ & $\mathrm{R} \$ 5,90$ & 3 \\
\hline Trigo $1 \mathrm{Kg}$ & $\mathrm{R} \$ 2,79$ & $R \$ 2,59$ & $R \$ 2,60$ & $\mathrm{R} \$ 2,40$ & $\mathrm{R} \$ 2,61$ & 8 \\
\hline $\begin{array}{l}\text { Acúcar } \\
\text { Refinado } 1 \mathrm{Kg}\end{array}$ & $\mathrm{R} \$ 1,89$ & $\mathrm{R} \$ 1,77$ & $\mathrm{R} \$ 1,69$ & $\mathrm{R} \$ 1,81$ & $\mathrm{R} \$ 1,95$ & 2 \\
\hline Sal $1 \mathrm{Kg}$ & $\mathrm{R} \$ 2,19$ & $\mathrm{R} \$ 2,23$ & $\mathrm{R} \$ 2,10$ & $\mathrm{R} \$ 2,45$ & $\mathrm{R} \$ 2,29$ & 1 \\
\hline Biscoito 330g & $R \$ 3,49$ & $\mathrm{R} \$ 3,73$ & $\mathrm{R} \$ 3,50$ & $R \$ 3,48$ & $\mathrm{R} \$ 3,45$ & 3 \\
\hline Óleo 900ml & $R \$ 2,59$ & $\mathrm{R} \$ 3,05$ & $\mathrm{R} \$ 3,23$ & $\mathrm{R} \$ 3,35$ & $\mathrm{R} \$ 3,79$ & 4 \\
\hline Vinagre $750 \mathrm{ml}$ & $\mathrm{R} \$ 2,41$ & $\mathrm{R} \$ 3,91$ & $\mathrm{R} \$ 3,18$ & $\mathrm{R} \$ 2,97$ & $R \$ 3,05$ & 1 \\
\hline $\begin{array}{l}\text { Amido de } \\
\text { Milho 500g }\end{array}$ & $R \$ 5,02$ & $R \$ 6,49$ & $R \$ 6,40$ & $\mathrm{R} \$ 6,10$ & $R \$ 6,18$ & 2 \\
\hline Manteiga 500g & $\mathrm{R} \$ 5,18$ & $\mathrm{R} \$ 5,99$ & $\mathrm{R} \$ 4,97$ & $\mathrm{R} \$ 5,25$ & $\mathrm{R} \$ 4,90$ & 2 \\
\hline Fubá 500g & $\mathrm{R} \$ 2,84$ & $\mathrm{R} \$ 1,19$ & $\mathrm{R} \$ 2,00$ & $\mathrm{R} \$ 2,55$ & $\mathrm{R} \$ 2,78$ & 2 \\
\hline $\begin{array}{l}\text { Extrato de } \\
\text { Tomate } 340 \mathrm{~g}\end{array}$ & $R \$ 3,20$ & $\mathrm{R} \$ 2,45$ & $\mathrm{R} \$ 2,55$ & $\mathrm{R} \$ 2,60$ & $R \$ 2,39$ & 2 \\
\hline Distância & 0 & $40 \mathrm{Km}$ & $83 \mathrm{Km}$ & $25 \mathrm{Km}$ & $39 \mathrm{Km}$ & \\
\hline
\end{tabular}

Após os ajustes, correção, validação e conferência das informações, o aplicativo Lista Fácil, realizou o cálculo das opções de compra apresentadas na Figura 11.

O cálculo foi realizado da seguinte forma: A função objetivo é calcular o menor custo da lista de produtos em que a quantidade de cada produto representa uma variável básica:

$$
\text { X1 = Arroz; X2 = Feijão; X3 = Macarrão; }
$$

X4 = Leite Integral; X5 = Café; X6 = Trigo; X7 = Açúcar Refinado; X8 = Sal; X9 = Biscoito; $x 10$ = Óleo; $\mathrm{X} 11$ = Vinagre; $\mathrm{X} 12$ = Amido de Milho; X13 = Manteiga; X14 = Fubá; X15 = Extrato de Tomate;
Para cada estabelecimento, foi aplicada a função com as restrições como apresentado na sequência.

Esse é o Exemplo do Supermercado Kawakami, em que foi atribuído a cada variável de quantidade o valor unitário do produto.

A função objetivo de minimização de custo é apresentada da seguinte forma:

Min $Z=11,80 \times 1+2.59 \times 2+2.17 \times 3+$ $2.89 \times 4+5,48 \times 5+2.79 \times 6+1.89 \times 7+2.19 \times 8+$ $3.49 \times 9+2.59 \times 10+2.41 \times 11+5.02 \times 12+$ $5.18 \times 13+2.84 \times 14+3.20 \times 15$ 
Sujeito a:

$$
\begin{aligned}
& X 1 \text { >= } ; \\
& X 2>=2 ; \\
& X 3>=3 ; \\
& X 4>=24 ; \\
& X 5>=3 ; \\
& X 6>=8 ; \\
& X 7>=2 ; \\
& X 8>=1 ; \\
& X 9>=3 ; \\
& X 10>=4 ; \\
& X 11>=1 ; \\
& X 12>=2 ; \\
& X 13>=2 ; \\
& X 14 \text { >= } 2 ; \\
& X 15>=2 ;
\end{aligned}
$$

No final do cálculo acrescenta-se o custo da distância sobre o resultado obtido no cálculo.

Foi utilizado para a comparação dos resultados do aplicativo Lista Fácil o Software
LINDO (LINDO, 2014), que é uma ferramenta usada para realizar cálculos de apoio a tomada de decisão usado por milhares de empresas em todo o mundo para maximizar o lucro e minimizar o custo das decisões que envolvem o planejamento da produção, transporte, finanças, alocação de portfólio, orçamento de capital, de mistura, programação, estoques, recursos alocação e muito mais.

Utilizando o Software LINDO, é calculada a minimização para cada estabelecimento e é verificado que os resultados conferem com os exibidos pelo aplicativo Lista Fácil. Os resultados são apresentados na Figura 9 para o Supermercado Kawakami de Bastos/SP, na Figura 10 para o Supermercado Avenida de Tupã/SP e na Figura 11 para o Supermercado Avenida da cidade de Rancharia/SP. 


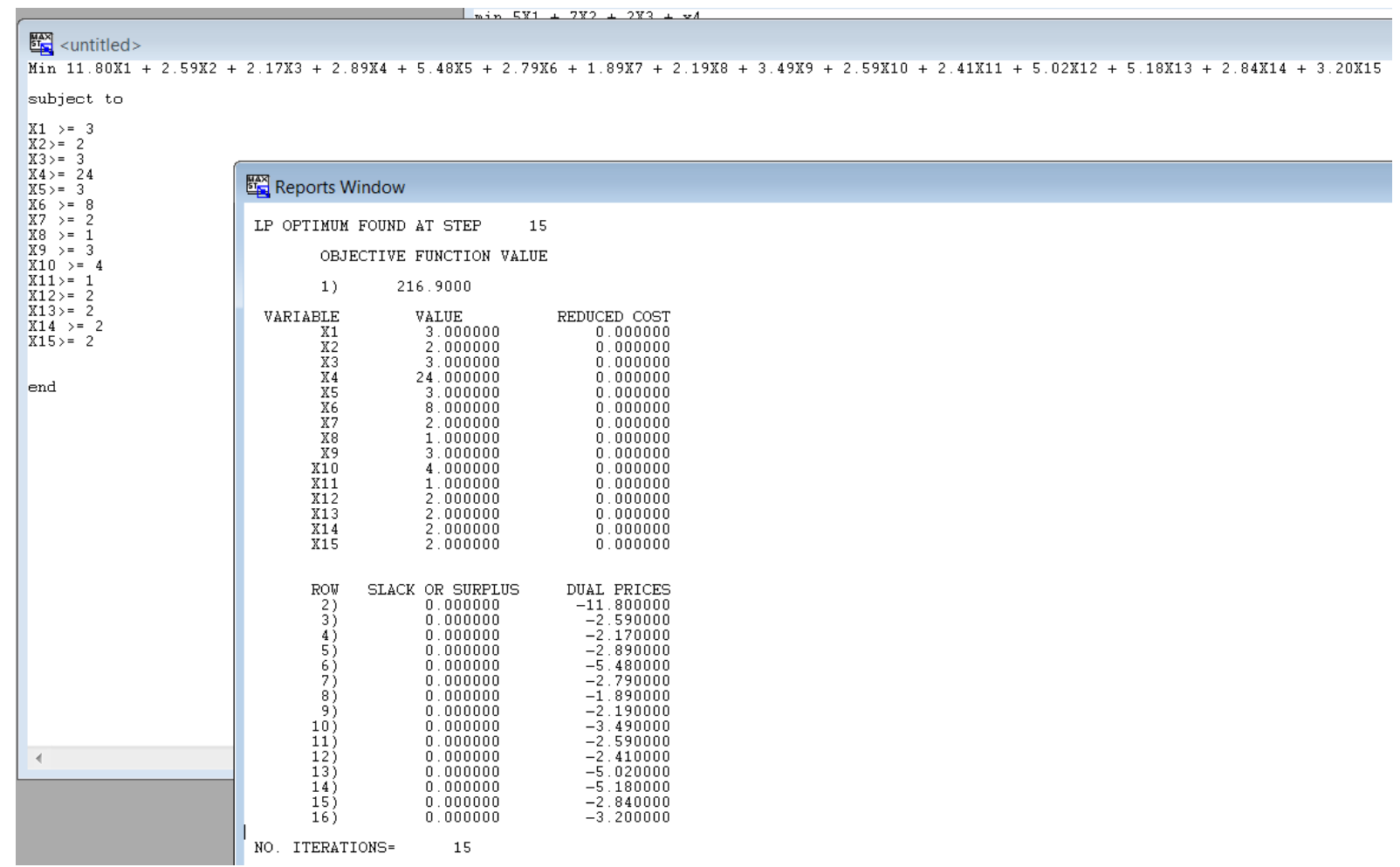

Figura 9. Resultado do Cálculo para o Supermercado Kawakami. Em a) tem-se a fórmula que foi criada para o cálculo, com as informações do Supermercado Kawakami preenchidas na função objetivo e suas restrições. ; em b) tem-se o resultado do cálculo da função que teve como retorno o valor de 216,90;

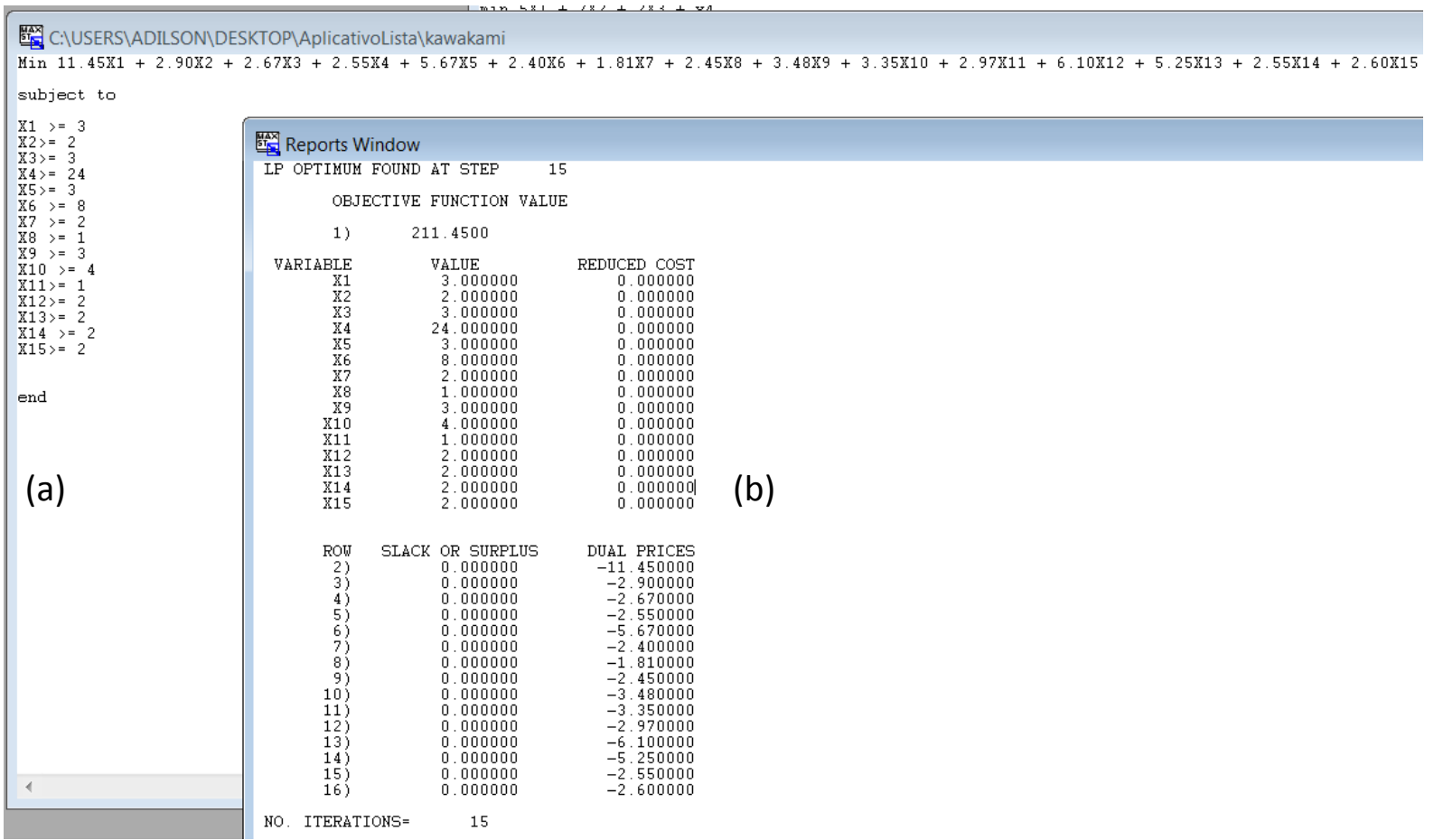

Figura 10. Resultado do Cálculo para o Supermercado Avenida - Tupã SP. Em a) tem-se a fórmula que foi criada para o cálculo, com as informações do Supermercado Avenida de Tupã/SP preenchidas na função objetivo e suas restrições; em b) tem-se o resultado do cálculo da função que teve como retorno o valor de 211,45 ; 


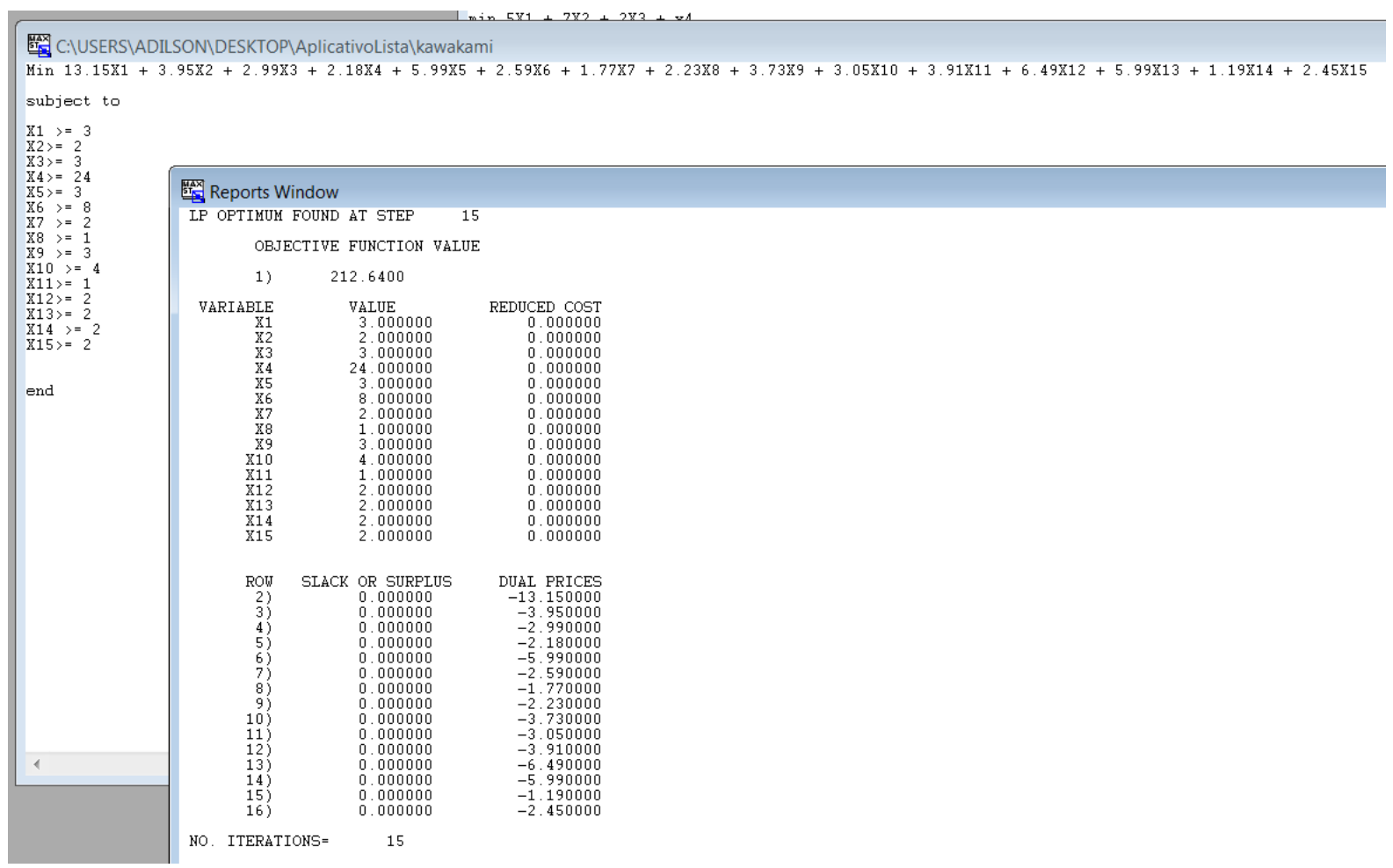

Figura 11. Resultado do Cálculo para o Supermercado Avenida - Rancharia /SP. Em a) tem-se a fórmula que foi criada para o cálculo com as informações do Supermercado Avenida de Rancharia/SP preenchidas na função objetivo e suas restrições; em b) tem-se o resultado do cálculo da função que teve como retorno o valor de 212,64.

Pode-se verificar que os mesmos resultados calculados com o uso do Software Lindo são apresentados pelo aplicativo Lista Fácil. Sobre o resultado do custo retornado é somada a distância para o estabelecimento. $\mathrm{Na}$ Figura 11 observa-se $\mathrm{o}$ resultado retornado pelo aplicativo Lista Fácil. Apesar do custo no Supermercado Avenida de Tupã ser menor, a distância é maior em relação ao Supermercado Kawakami de Bastos/SP.

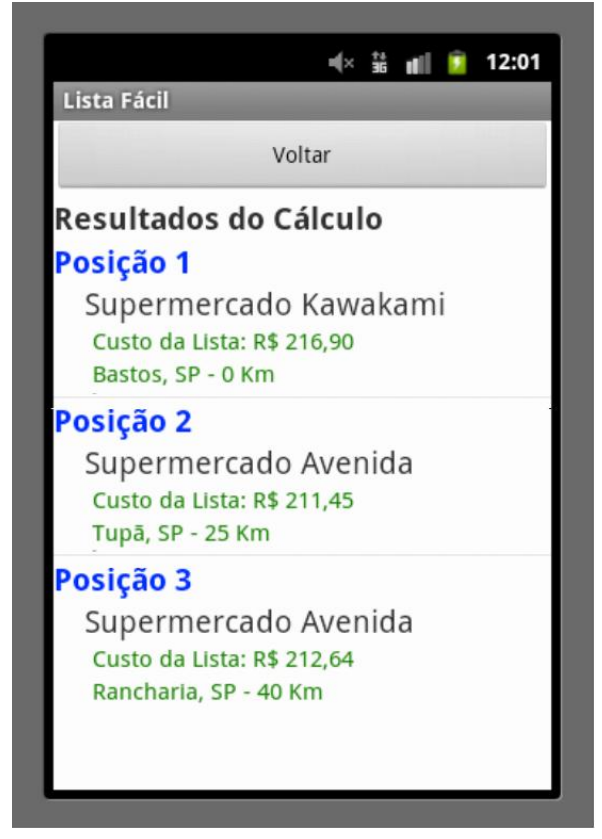

Figura 12. Resultado do cálculo realizado com base nos produtos da Tabela 2 . 


\section{CONSIDERAÇÕES FINAIS}

Com o desenvolvimento deste trabalho, pôde-se mostrar a importância que, com a troca de informações, por meio de dispositivos móveis, e o uso da inteligência coletiva é possível realizar economia na compra de produtos.

Pôde-se levar também em consideração a utilidade prática do aplicativo que pode auxiliar e ser muito útil para realização de economia no processo de compra.

Algumas modificações podem ser desenvolvidas para aprimorar este trabalho, como por exemplo, ajustar o cálculo de menor custo considerando compras parciais da lista de produtos em locais diferentes, agrupando as compras para não se ter gasto maior e assim aumentar a economia nas compras.

\section{REFERÊNCIAS}

BBC BRASIL. Entenda por que a inflação preocupa no Brasil. 10 abr. 2013. Disponível em

<http://www.bbc.co.uk/portuguese/noticias/ 2013/04/130409_inflacao_

entenda_lgb.shtml>. Acesso em: $05 \mathrm{fev}$. 2014.

BOA LISTA. Lista de compras, preços, ofertas 2014. Disponível em: <https://www.boalista.com.br>. Acesso em: 18 fev. 2014.

BUENO, M. A influência dos smartphones no processo de compra. Revista E-commerce Brasil, ano 3, ed. 16, p.44, ago. 2013.
Disponível em:

<http://www.ecommercebrasil.com.br/revist a/?edition_id=16\&folhear=true\&pageNumbe $r=44>$. Acesso em: 21 nov. 2013.

BUSCAPÉ COMPANY. Meu carrinho. 2014. Disponível em: <https://www.buscapecompany.com/pt/mar cas-meucarrinho.htm>. Acesso em: 31 maio 2014.

CECCATTO, T. J. Aplicativo de comparação e divulgação de produtos em tempo real para dispositivos móveis. 2012. Trabalho (Conclusão do Curso) - Universidade do Planalto Catarinense, Lajes, SC.

G1. Aplicativo gratuito compara preços de supermercados. 2012. Disponível em:

<http://g1.globo.com/pr/parana/noticia/201 2/05/aplicativo-gratuito-compara-precos-desupermercados-em-curitiba.html>. Acesso em: 02 fev. 2014.

GIZMODO BRASIL. No Brasil, smartphones Android dominam $90 \%$ do mercado e Windows Phone tenta ultrapassar iOS. 23 ago. 2013. Disponível em: <http://gizmodo.uol.com.br/smartphonesbrasil-2q2013/>. Acesso em: 08 mai. 2014.

GOOGLE PLAY. Aplicativo Touch Economia. $2014 . \quad$ Disponível em: <https://play.google.com/store/apps/details ?id=getcs.toqueeconomia $>$. Acesso em: 15 abr. 2014.

HOWE, J. $\mathbf{O}$ poder das multidões: porque a força da coletividade está remodelando o futuro dos negócios. 2. ed. Rio de Janeiro: 2009.

$\mathrm{ICl}$. O seu dinheiro vale mais com o Touch Economia. 2012. Disponível em: $<$ http://www.ici.curitiba.org.br/noticias/seudinheiro-vale-mais-com-o-toucheconomia/688>. Acesso em: 31 maio 2014. 
LACHTERMACHER, G. Pesquisa operacional na tomada de decisões. Rio de Janeiro: Campus, 2002.

LECHETA, R. R. Google Android: aprendar a criar aplicações para dispositivos móveis com Android SDK. 2. Ed. São Paulo: Novatec Editora, 2010.

LÉVY, P. A inteligência coletiva: por uma antropologia do ciberespaço. 5. ed. São Paulo: Loyola, 2007.

LINDO. Software for Integer Programming, Linear Programming, Nonlinear Programming, Stochastic Programming, Global Optimization. Disponível em: <http://www.lindo.com>. Acesso em: 10 maio 2014.

OKIISHI, T. F.; SOUZA, L. F. R. Método Simplex aplicado à minimização dos custos de transporte de uma rede de farmácias. Revista Eletrônica de Educação e Ciência (REEC), v.03, n.01, 2013. Disponível em: <http://www.fira.edu.br/revista/vol3_num1_ pag15.pdf>. Acesso em: 22 nov. 2013.

\section{OUR MOBILE PLANET. Nosso Planet Mobile}

Brasil: como entender o usuário de celular, primeiro trimestre de 2013. Disponível em: <http://ow.ly/m9C0K>. Acesso em: 25 nov. 2013.

TANENBAUM, A. S. Redes de computadores. Rio de Janeiro: Campus, 2003.

TECHTUDO. 0 que é Smartphone e para que serve? 28 dez. 2011. Disponível em: <http://www.techtudo.com.br/artigos/notici a/2011/12/o-que-e-smartphone-e-para-queserve.html>. Acesso em: 18 fev. 2014.

ZXING. Multi-format 1D/2D barcode image processing library with clients for Android, Java. Disponível em: <https://code.google.com/p/zxing/>.

Acesso em: 18 fev. 2014. 